\title{
Eyemote - towards context-aware gaming using eye movements recorded from wearable electrooculography
}

\section{Conference Paper}

Author(s):

Bulling, Andreas; Roggen, Daniel; Tröster, Gerhard

Publication date:

2008

Permanent link:

https://doi.org/10.3929/ethz-a-005705469

Rights / license:

In Copyright - Non-Commercial Use Permitted 


\title{
EyeMote - Towards Context-Aware Gaming Using Eye Movements Recorded From Wearable Electrooculography
}

\author{
Andreas Bulling, Daniel Roggen and Gerhard Tröster \\ ETH Zurich, Wearable Computing Laboratory \\ bulling@ife.ee.ethz.ch
}

\begin{abstract}
Physical activity has emerged as a novel input modality for so-called active video games. Input devices such as music instruments, dance mats or the Wii accessories allow for novel ways of interaction and a more immersive gaming experience. In this work we describe how eye movements recognised from electrooculographic (EOG) signals can be used for gaming purposes in three different scenarios. In contrast to common video-based systems, EOG can be implemented as a wearable and light-weight system which allows for long-term use with unconstrained simultaneous physical activity. In a stationary computer game we show that eye gestures of varying complexity can be recognised online with equal performance to a state-of-the-art video-based system. For pervasive gaming scenarios, we show how eye movements can be recognised in the presence of signal artefacts caused by physical activity such as walking. Finally, we describe possible future context-aware games which exploit unconscious eye movements and show which possibilities this new input modality may open up.
\end{abstract}

\section{Introduction}

The recognition of user activity has turned out to play an important role in the development of today's video games. Getting the player physically involved in the game provides a more immersive experience and a feeling of taking direct part rather than just playing as an external beholder. Motion sensors have already been implemented to recognise physical activity: Game controllers such as music instruments, guns, dance mats or the Wii accessories make use of different sensors to open up a whole new field of interactive game applications. However, in pervasive settings, the use of physical activity may not be sufficient or not always be desired. Furthermore, cognitive aspects like user attention and intentionality remain mainly unexplored despite having a lot of potential for gaming scenarios. Therefore, alternative input modalities need to be developed which enable new gaming scenarios, are unobtrusive and can be used in public without affecting privacy.

A lot of information about the state of the user can be found in the movement of the eyes. Conscious eye movement patterns provide information which can be used to recognise user activity such as reading [1]. Explicit eye gestures performed by the player may be used for direct game input. Unconscious eye movements are related to cognitive processes such as attention [2], saliency determination [3], visual memory [4] and perceptual learning [5]. The analysis of these movements may eventually allow novel game interfaces to deduce information on user activity and context not available with current sensing modalities. In this paper we describe how Electrooculography (EOG) can be used for tracking eye movements in stationary and pervasive game scenarios. Additionally, we discuss which possibilities unconscious eye movements may eventually provide for future gaming applications. 


\section{Related Work}

\subsection{Eye-based Human-Computer Interaction}

Eye tracking using vision for human-computer interaction has been investigated by several researchers. Most of their work has focused on direct manipulation of user interfaces using gaze (e.g. [6,7]). Drewes et al. proposed to use eye gestures to implement new ways of human-computer interaction [8]. They showed that gestures are robust to different tracking accuracies and calibration shift and do not exhibit the "Midas touch" problem [9].

\subsection{Eye Movements and Games}

Smith et al. studied eye-based interaction for controlling video games across different genres, a first-person shooter, a role-playing game and an action/arcade game [10]. By comparing eye-based and mouse-based control they found that using an eye tracker can increase the immersion and leads to a stronger feeling of being part of the game. In a work by Charness et al., expert and intermediate chess players had to choose the best move in five different chess positions with their eyes [11]. Based on the analysis of eye motion they found that more experienced chess players showed eye movement patterns with a higher selectivity depending on chess piece saliency. Lin et al. developed a game interface using eye movements for rehabilitation [12]. They reported that the subjects' eyes became more agile which may allow for specific applications to help people with visual disabilities.

\subsection{EOG-based Interfaces}

Several studies show that EOG can be implemented as an easy to operate and reliable interface. Eye movement events detected in EOG signals such as saccades, fixations and blinks have been used to control robots [13] or a wearable system for medical caregivers [14]. Patmore et al. described a system that provides a pointing device for people with physical disabilities [15]. All of these systems use basic eye movements or eye-gaze direction but they do not implement movement sequences which provide a more versatile input modality for gaming applications.

\section{Wearable Electrooculography}

\subsection{Eye Movement Characteristics}

The eyes are the origin of an electric potential field which is usually described as a dipole with its positive pole at the cornea and its negative pole at the retina. This so-called corneoretinal potential (CRP) is the basis for a signal measured between two pairs of electrodes commonly placed above and below, and on the left and right side of the eye, the so-called Electrooculogram (EOG).

If the eyes move from the centre position towards the periphery, the retina approaches one of the electrodes while the cornea approaches the opposing one. This results in a change in the electric potential. Inversely, eye movements can be tracked by analysing these changes in the EOG signal.

In the human eye, only a small central region of the retina, the fovea, is sensitive enough for most visual tasks. This requires the eyes to move constantly as only small parts of a scene can be perceived with high resolution. Simultaneous movements of both eyes in the same direction are called saccades. Fixations are static states of the eyes during which gaze is held at a specific location. 


\subsection{EOG Data Recording}

EOG, in contrast to well established vision-based eye tracking ${ }^{1}$, is measured with body-worn sensors, and can therefore be implemented as a wearable system. In earlier work we described how unobtrusive EOG recordings can be implemented with a light-weight and potentially cheap device, the wearable eye tracker [16]. The device consists of Goggles with integrated dry electrodes and a signal processing unit called Pocket with a Bluetooth and a MMC module. This unit can also be worn on the body, e.g. in a cloth bag fixed to one of the upper arms. Four EOG electrodes are arranged around the left eye and mounted in such a way as to achieve permanent skin contact. Finally, a 3-axis accelerometer and a light sensor are attached to the processing unit with the latter pointing forward in line of incident light (see Figure 1). The system weights $208 \mathrm{~g}$ and allows for more than 7 hours of mobile eye movement recording.
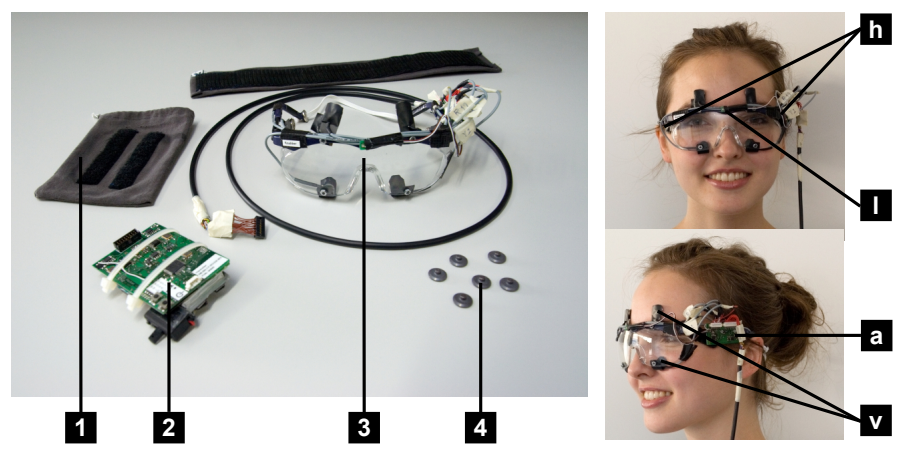

Fig. 1. Components of the EOG-based wearable eye tracker: armlet with cloth bag (1), the Pocket (2), the Goggles (3) and dry electrodes (4). The pictures to the right show the Goggles worn by a person with the positions of the two horizontal (h) and vertical (v) electrodes, the light sensor (l) and the accelerometer (a).

\subsection{EOG Signal Processing}

To detect complex eye gestures consisting of several distinct movements from EOG signals the stream of saccades needs to be processed and analysed in a defined sequence [16]. This detection has several challenges with the most important one being to reliably detect the saccade events in the continuous vertical and horizontal EOG signal streams. Another challenge are the various types of signal artefacts which hamper the signal and can affect eye gesture recognition. This involves common signal noise, but also signal artefacts caused by physical activity which need to be removed from the signal. The characteristics of blinks are very similar to those of vertical eye movements, therefore they may need to be removed from the signal. However, for certain applications, blinks may also provide a useful input control, thus only reliable detection is required.

\footnotetext{
${ }^{1}$ With "eye tracking" we understand the recording and analysis of eye motion in contrast to "gaze tracking" which deals with tracking eye-gaze direction.
} 
The output of the wearable eye tracker consists of the primitive controls left, right, up, down and diagonal movements (see Figure 2), blinks (conscious and unconscious), saccades and fixations. In addition, the system provides the following lowlevel signal characteristics and additional sensor inputs: EOG signal amplitudes (horizontal, vertical), timing of eye movement events, relative gaze angle, head movement (3-axis acceleration signal) and level of ambient light. Finally, the device can provide high-level contextual information, e.g. on user activity [1] or eventually the user's cognitive load or attention.

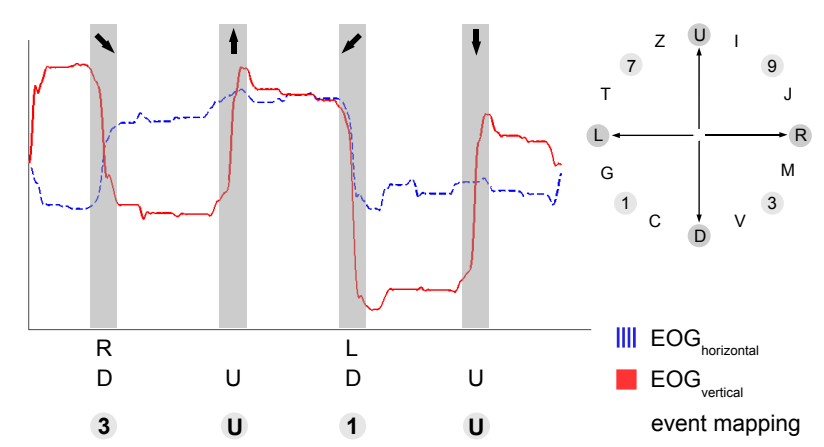

Fig. 2. Eye movement event encoding from horizontal and vertical EOG signals for gesture $3 \mathrm{U} 1 \mathrm{U}$ : Windows marked in grey with distinct eye movement events detected in the horizontal and vertical signal component and final mapping to basic (U) and diagonal $(3,1)$ movements. The top right corner shows the symbols representing the possible directions for eye movement encoding.

\section{Application Scenarios}

In this section we describe how eye movements recorded from wearable EOG can be used for different game scenarios. We first focus on stationary and pervasive settings involving direct game control. Afterwards, we give an outlook to future work and discuss which possibilities the analysis of unconscious eye movements may eventually provide and which novel gaming applications this may enable.

\subsection{Stationary Games}

The first scenario considers interactive games which are played in stationary settings with constrained body movements. These types of gaming applications are typically found at home, e.g. while sitting in front of a console in the living room or at the computer in the workroom. As the player does not perform major body movements, the weight and size of a game controller is not a critical issue. Instead, aspects such as natural and fast interaction are of greater importance.

To assess the feasibility of using the wearable eye tracker as an input device in stationary settings we investigated a simplified game consisting of eight different levels. In each game level, subjects had to perform a defined eye gesture consisting of a changing number of consecutive eye movements (see Table 1). The gestures in the experiment were selected to be of increasing complexity. For future stationary 


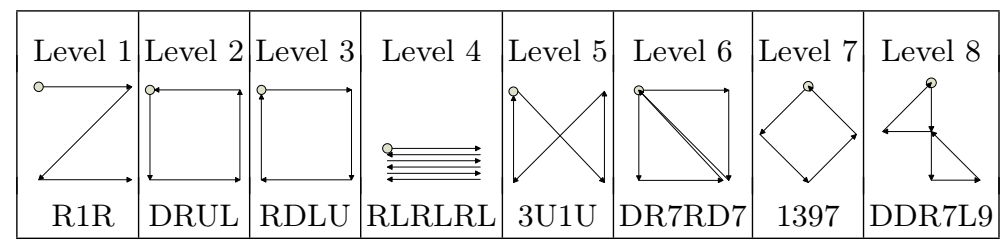

Table 1. Eye gestures of increasing complexity and their string representations used in the eight levels of the computer game (cf. Figure 2). The grey dot denotes the start and the arrows the order and direction of each eye movement.

games, eye gestures may for example be used for direct user feedback or ingame task control.

Each eye gesture was to be repeatedly performed as fast as possible until the first successful try. If a wrong eye movement was recognised, i.e. one which was not part of the expected gesture, the level was restarted and a penalty was rewarded on the game score. Once the whole eye gesture was successfully completed the next game level showing the next gesture was started. For each level, the number of wrong and correct eye movements as well as the required time were recorded.

The subjects had to perform three runs with all game levels being played in each run. The first was a test run to introduce the game and calibrate the system for robust gesture recognition. In two subsequent runs the subjects played all levels of the game once again. At the end of the experiment, the subjects were asked on their experiences on the procedure in a questionnaire.

The experiment was conducted using the wearable eye tracker, a standard desktop computer and a 17 " flat screen with a resolution of $1024 \times 768$ pixels. The subjects were sitting in front of the screen facing its centre with movements of the head and the upper body allowed at any time. The expected eye movement order and their directions were shown as blue arrows with grey dots denoting the start and end point of a movement (see Figure 3).

\begin{tabular}{|l|c|c|c|c|c|c|c|c|c|c|c|c|}
\hline Gesture & \multicolumn{9}{|c|}{ Accuracy [\%] } \\
& $\mathbf{S 1 ( m )}$ & $\mathbf{S 2 ( f )}$ & $\mathbf{S 3 ( f )}$ & $\mathbf{S 4}(\mathbf{m})$ & $\mathbf{S 5}(\mathbf{m})$ & $\mathbf{S 6}(\mathbf{m})$ & $\mathbf{S 7}(\mathbf{m})$ & $\mathbf{S 8}(\mathbf{m})$ & $\mathbf{S 9}(\mathbf{m})$ & $\mathbf{S 1 0}(\mathbf{m})$ & $\mathbf{S 1 1}(\mathbf{m})$ \\
\hline R1R & 88 & 69 & 100 & 100 & 69 & 100 & 100 & 100 & 100 & 90 & 66 \\
DRUL & 100 & 71 & 100 & 100 & 86 & 100 & 100 & 90 & 100 & 100 & 90 \\
RDLU & 100 & 100 & 100 & 100 & 100 & 88 & 100 & 90 & 100 & 100 & 100 \\
RLRLRL & 100 & 100 & 95 & 93 & 100 & 100 & 92 & 82 & 100 & 89 & 88 \\
3U1U & 79 & 100 & 90 & 100 & 100 & 100 & 75 & 100 & 90 & 90 & 92 \\
DR7RD7 & 90 & 95 & 78 & 71 & 90 & 93 & 88 & 75 & 96 & 80 & 100 \\
1379 & 73 & 90 & 100 & 83 & 88 & 81 & 100 & 76 & 85 & 89 & 100 \\
DDR7L9 & 95 & 91 & 93 & 71 & 89 & 77 & 100 & 73 & 76 & 76 & 100 \\
\hline Average & $\mathbf{9 1}$ & $\mathbf{8 9}$ & $\mathbf{9 5}$ & $\mathbf{9 0}$ & $\mathbf{9 0}$ & $\mathbf{9 2}$ & $\mathbf{9 4}$ & $\mathbf{8 6}$ & $\mathbf{9 3}$ & $\mathbf{8 9}$ & $\mathbf{9 2}$ \\
\hline
\end{tabular}

Table 2. Accuracy for the different gestures for each individual subject without test run. The accuracy gives the ratio of eye movements resulting in a correct gesture to the total number of eye movements performed. The table also shows the subjects' gender (f: female, m: male). 


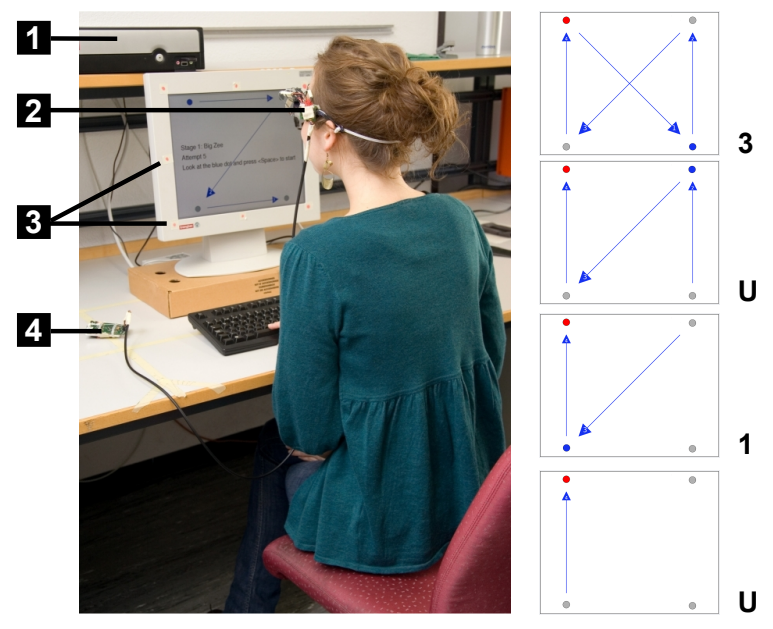

Fig. 3. Experimental setup consisting of a desktop computer running the game (1), the Goggles (2), a flat screen with red dots used for calibration (3) and the Pocket (4). The screenshots on the right show the sequence of eye movements and the generated event symbols for gesture $3 \mathrm{U} 1 \mathrm{U}$ (from top to bottom). The red dot denotes the start of the gesture and the blue dot its end point. Blue arrows indicate the order and the direction of each expected eye movement.

Results We collected data from 11 subjects - 2 female and 9 male - aged 24 to 64. The results for each individual subject only show a small range of different accuracies (see Table 2). The results were calculated using data from the second and the third run as the first one was for testing. The accuracy was calculated as the ratio of eye movements resulting in a correct gesture to the total number of eye movements performed in the level. The highest result is $95 \%$ (subject 3) while the worst result is for subject 8 , with an accuracy of $86 \%$. It can be seen from the table that performance does not correlate to the gender of the subject.

Table 3 shows the average performance over all subjects, i.e. the time and the accuracy to perform each of the eight gestures. $T_{T}$ denotes the total time the subjects spent trying to complete each of the gestures; the success time $T_{S}$ only measures the time spent on all successful attempts.

Table 4 shows the average response time $T_{R}$ required to perform five gestures in comparison to a video-based system [8]. $T_{R}$ was calculated from $T_{T}$ to take the different experimental setups into account (see [16] for details).

Figure 4 shows the average accuracy for different eye movements and its increase during the three experimental runs.

\subsection{Pervasive Games}

The second scenario considers pervasive games which are not constrained in terms of the players' body movements and/or not restricted to a certain location [17]. These games may therefore either be played indoors in front of a console, in combined virtual and physical environments or in daily life settings (e.g. role plays in natural environments). They require wearable equipment which needs to be light-weight and low-power to allow for unobtrusive and autonomous (long-term) use. Furthermore, pervasive games allow potentially more complex multi-modal and ubiquitous 


\begin{tabular}{|l|c|c|c|}
\hline Gesture & $\boldsymbol{T}_{\boldsymbol{T}}[\boldsymbol{m s}]$ & $\boldsymbol{T}_{\boldsymbol{S}}[\boldsymbol{m s}]$ & Accuracy [\%] \\
\hline R1R & 3370 & 2890 & 85 \\
DRUL & 4130 & 3490 & 90 \\
RDLU & 3740 & 3600 & 93 \\
RLRLRL & 6680 & 5390 & 90 \\
3U1U & 4300 & 3880 & 89 \\
DR7RD7 & 12960 & 5650 & 83 \\
1379 & 6360 & 3720 & 84 \\
DDR7L9 & 25400 & 5820 & 83 \\
\hline
\end{tabular}

Table 3. Average performance and accuracy for the different gestures over all subjects without test run. The accuracy is the ratio of eye movements resulting in a correct gesture to the total number of eye movements performed until success. $T_{T}$ is the total time spent to complete the gesture and $T_{S}$ the success time spent only on successful attempts.

\begin{tabular}{|l|c|c|}
\hline Gesture & \multicolumn{2}{|c|}{$\boldsymbol{T}_{\boldsymbol{R}}[\boldsymbol{m s}]$} \\
& EOG & Video \\
\hline \hline DRUL & 1520 & 1818 \\
RDLU & 1630 & 1905 \\
RLRLRL & 2860 & 3113 \\
3U1U & 1940 & 2222 \\
DR7RD7 & 5890 & 3163 \\
\hline
\end{tabular}

Table 4. Average response time $T_{R}$ required to perform five different eye gestures over all subjects without initial test run in comparison to a video-based system.

interaction with(in) the environment, for example with combined hand and eye gestures. Eye gestures in pervasive games may provide two functions: (1) they allow the player to be immersed in the environment, especially when/if combined with head up displays and (2) at the same time allow for privacy, since eye gestures are not likely to be noticed as it is the case for body gestures.

As EOG is measured with body-worn sensors, body motion causes artefacts in the signals and affects eye movement detection. However, EOG can still be used in mobile settings. To show the feasibility of using the wearable eye tracker with simultaneous physical activity we carried out an experiment which involved subjects to perform different eye movements on a head-up display (HUD) while standing and walking down a corridor. Walking is a common activity, thus serves well as a test bench for investigating how artefacts induced by body motion can be automatically compensated in EOG signals. We evaluated an adaptive median filter which first detects walking activity using the data from the acceleration sensor attached to the Goggles. If walking is detected, the filter then continuously optimises its parameters to the walking pace to reduce signal artefacts.

The experiment was done using the wearable eye tracker, a standard laptop, a SV-6 head-up display from MicroOptical with a resolution of $640 \times 480$ pixels mounted to the Goggles frame and a wearable keyboard Twiddler2 (see Figure 5). The laptop was used to run the experiment software. During the experiments, the laptop was worn in a backpack in order not to constrain the subjects during walking. As the experimental assistant did not have control over the system, once the experiment 


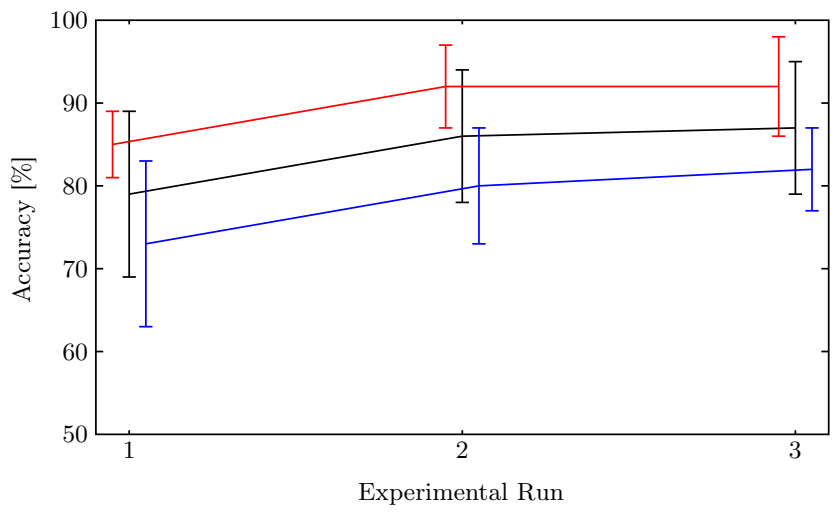

Fig. 4. Plot of distinct eye movement performance with standard deviation over all subjects for each experimental run. The red line shows the accuracy for movements in the basic directions (U,D,R,L), the blue one for diagonal movements $(1,3,7,8)$ and the black plot the average over all movements.

was started, the Twiddler 2 was needed to allow the subjects to control the software and start the different recordings.

The subjects were first trained on the game from the first experiment using the laptop screen. Once the game was finished, the HUD was attached to start the second experiment. The subjects performed three runs each consisting of different visual tasks while standing and walking down a corridor. Similar to the first experiment, for each of these tasks, the sequence and direction of the expected eye movements were indicated on the HUD as arrows and a moving red dot. The subjects were asked to concentrate on their movements and fixate this dot permanently.

The first run was carried out as a baseline case with fixations on the centre of the screen and large saccades without using the HUD. In two subsequent runs the subjects were asked to perform different sequences of eye movements on the HUD while standing and walking: The second run only contained simple movements in vertical and horizontal direction. The third run also included additional movements along the diagonals (cf. Figure 2).

Results We recorded 5 male subjects between the age of 21 and 27 totalling roughly 35 minutes of recording with walking activity accounting for about 22 minutes. To assess a relative performance measure, we did a comparison to a standard median filter with fixed window size.

Figure 6 shows a boxplot for the total number of detected saccades in the horizontal EOG signal component of run 3. Each box summarises the statistical properties of the data of the 5 subjects: The horizontal red lines in each box indicates the median and the upper and lower quartiles. The vertical dashed lines indicate the data range, points outside their ends are outliers. Boxes are plotted for the following cases: stationary and raw signal, stationary and fixed median filter, stationary and adaptive filter, walking and raw signal, walking and fixed median filter, walking and adaptive filter. The single solid horizontal line indicates the expected number of saccades defined by the experimental procedure.

What can be seen from the figure is that in the stationary case, both filters perform equally well. During walking, however, significant differences can be recognised: The raw recordings show about eight times more detected saccades than in the stationary case. As the number of expected eye movements was constrained by the software 

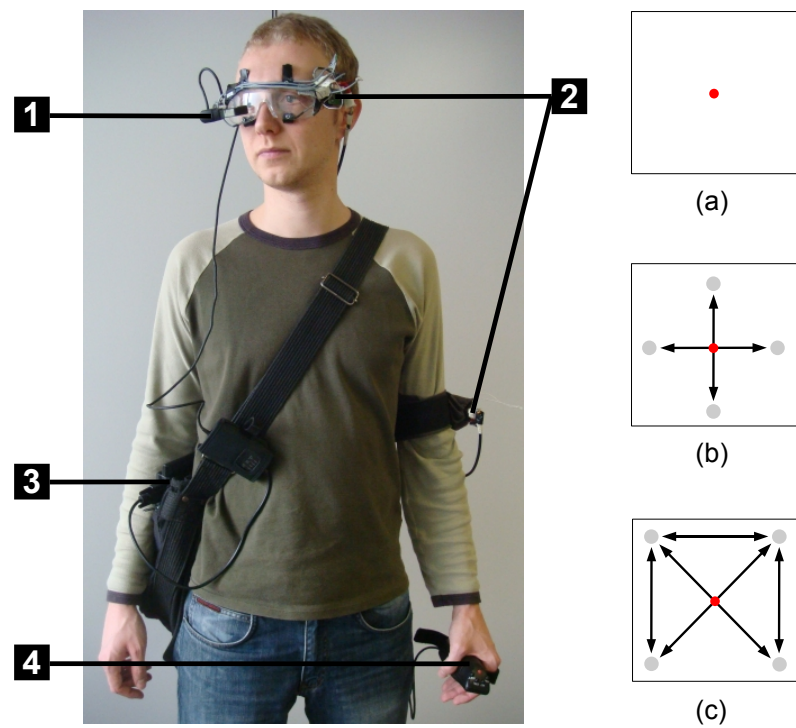

(a)

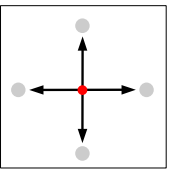

(b)

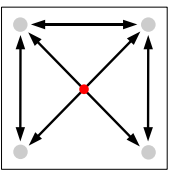

(c)

Fig. 5. Experimental setup consisting of a head-up display (1), the wearable eye tracker (2), a laptop (3) and a Twiddler2 (4). The screenshots on the right show the different eye movements performed in the three runs: Fixations on the centre of the screen (a), simple movements in vertical and horizontal direction (b) and additional movements along the diagonals (c) (cf. Figure 2). The red dots in the centre denote the start; arrows indicate the directions of the movements.

these additionally detected saccades can be considered signal artefacts caused by walking. While the median filter with a fixed window size fails in removing these artefacts, the adaptive filter still performs well. This shows that signal artefacts caused by motion can be cancelled, thereby enabling the use of EOG-based game interfaces in pervasive gaming scenarios.

\subsection{Future Context-Aware Games}

Given the success of the new input controllers of today's active video games, future games will probably see more natural and more sophisticated interaction. These may increasingly take place in everyday scenarios with multi-modal input, several people being involved in the same game and a high level of collaboration. In terms of eye movements as an input modality, game interfaces based on direct input will probably remain an important focus of research [18]. However, additional information related to the underlying cognitive processes and the user's context may open up new possibilities for game developers and players.

Inducing Flow and Optimal Game Experience Based on eye movement analysis, future games may be aware of the user's cognitive load, and adapt the individual gaming experience accordingly [19]. In particular, such games may increase the demand on the user when his cognitive load is assessed as being too weak, whereas demand may be decreased if cognitive load is recognised as being too high. This may enable the player to keep experiencing the feeling of full involvement and energised focus characteristic of the optimal experience, also known as 


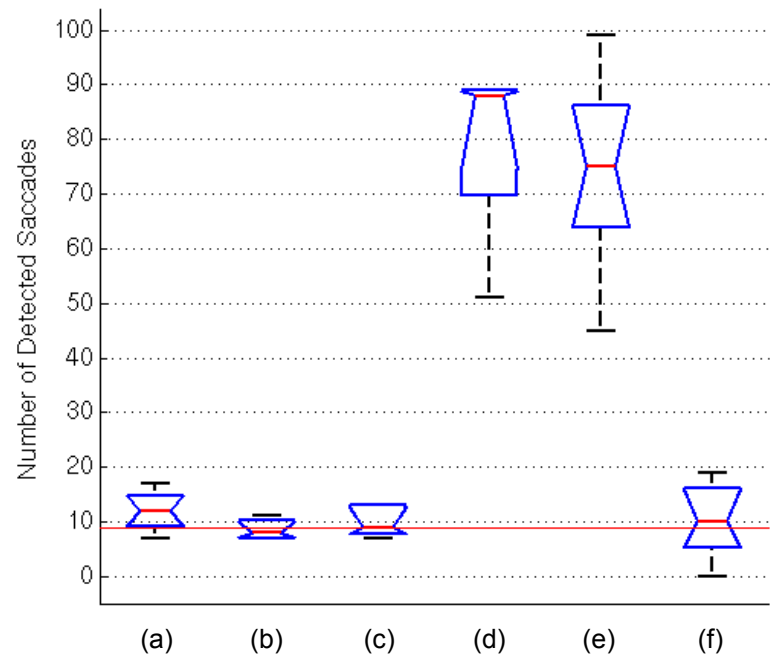

Fig. 6. Boxplot for the total number of detected saccades in the horizontal EOG signal component of run 3 with fixed thresholds over all subjects: stationary/raw (a), stationary/fixed median filter (b), stationary/adaptive filter (c), walking/raw (d), walking/fixed median filter (e), walking/adaptive filter (f). Horizontal red lines in each box indicate the lower quartile, median and upper quartile; dashed vertical lines show the data range; outliers are given as red crosses; the single solid horizontal line indicates the expected number of saccades.

flow [20]. In a collaborative game scenario this would allow to distinguish players with different game experience and adapt the game difficulty for a more balanced game experience.

Rehabilitation and Therapy Games Designers may also develop special games which require eye movements to be performed as exercises for medical purposes in rehabilitation or visual training. By using wearable EOG, these games could be brought to daily-life settings which would allow for permanent training independently from a special therapy at the doctor. The game exercises may be automatically adapted to the visual learning process derived from eye movement characteristics to optimise the training [5]. These games could be specifically optimised to fit the special requirements of children, elderly or even disabled people who still retain control of eye motion.

Context-Aware Gaming In a more general sense, future games may also provide new levels of context-awareness by taking into account different contextual aspects of the player. This context may comprise the player's physical activity, his location or mental state. Specific activities expressed by the eyes such as reading [1] could for example be used in games to adaptively scroll or zoom textual information. Context-aware games may also incorporate additional information derived from eye movements such as attention [21], task engagement [22] or drowsiness [23] to adapt to individual players. Other aspects of visual perception such as attention [2], saliency determination [3] and visual memory [4] may also enable new types of context-aware game interfaces not possible today. For collaborative games, 
this knowledge could be exchanged and combined into a common game context to integrate several players over potentially large geographical distances.

\section{Discussion and Conclusion}

In this work we have shown how a wearable electrooculographic system can be used for tracking eye movements in stationary and pervasive gaming scenarios. In the experiments, several subjects reported that the electrode placed below the eye was rather uncomfortable. In general, however, they did not feel constrained or distracted by wearing the goggles while gaming. To solve this issue, we are currently developing a new prototype of the system with improved electrode mounting. From the questionnaire we found that all subjects easily learned to use their eyes for direct game control. However, using explicit eye gestures was tiring and about $30 \%$ of the subjects had problems to stay concentrated during the game. Fatigue is an inherent problem also for input modalities such as hand gestures or speech. In pervasive settings, eye gestures outperform these modalities if the hands can not be used or if speech input is not possible.

Therefore, we believe EOG has a lot of potential for interactive gaming applications, in particular for those with unconstrained body movements. In contrast to videobased systems, EOG only requires light-weight equipment and allows for long-term use due to low power implementation. Unlike body movements, eye-based input allows for privacy which may prove extremely relevant in pervasive scenarios. Combined with a head-up display, EOG-based wearable eye tracking may eventually allow a more immersive game experience in outdoor environments. Information derived from unconscious eye movements may provide a more natural input modality for game control and future context-aware games. Thus, wearable EOG may become a powerful measurement technique for game designers and may pave the way for novel video games not possible so far.

\section{References}

1. A. Bulling, J. A. Ward, H.-W. Gellersen and G. Tröster. Robust Recognition of Reading Activity in Transit Using Wearable Electrooculography. In Proc. of the 6th International Conference on Pervasive Computing (Pervasive 2008), pages 19-37, 2008.

2. T. Selker. Visual attentive interfaces. BT Technology Journal, 22(4):146-150, 2004.

3. J. M. Henderson. Human gaze control during real-world scene perception. Trends in Cognitive Sciences, 7(11):498-504, 2003.

4. D. Melcher and E. Kowler. Visual scene memory and the guidance of saccadic eye movements. Vision Research, 41(25-26):3597-3611, 2001.

5. M. M. Chun. Contextual cueing of visual attention. Trends in Cognitive Sciences, 4(5):170-178, 2000.

6. S. Zhai, C. Morimoto and S. Ihde. Manual and gaze input cascaded (MAGIC) pointing. In Proc. of the SIGCHI Conference on Human Factors in Computing Systems (CHI 1999), pages 246-253, 1999.

7. P. Qvarfordt and S. Zhai. Conversing with the user based on eye-gaze patterns. In Proc. of the SIGCHI Conference on Human Factors in Computing Systems (CHI 2005), pages 221-230, 2005. 
8. H. Drewes and A. Schmidt. Interacting with the Computer Using Gaze Gestures. In Proc. of the 11th International Conference on Human-Computer Interaction (INTERACT 2007), pages 475-488, 2007.

9. R. J. K. Jacob. What you look at is what you get: eye movement-based interaction techniques. In Proc. of the SIGCHI Conference on Human Factors in Computing Systems (CHI 1990), pages 11-18, 1990.

10. J. D. Smith and T. C. N. Graham. Use of eye movements for video game control. In Proc. of the International Conference on Advances in Computer Entertainment Technology (ACE 2006), pages 20-27, 2006.

11. N. Charness, E. M. Reingold, M. Pomplun and D. M. Stampe. The perceptual aspect of skilled performance in chess: Evidence from eye movements. Memory and Cognition, 29:1146-1152(7), 2001.

12. C.-S. Lin, C.-C. Huan, C.-N. Chan, M.-S. Yeh and C.-C. Chiu. Design of a computer game using an eye-tracking device for eye's activity rehabilitation. Optics and Lasers in Engineering, 42(1):91-108, 2004.

13. W. S. Wijesoma, Kang S. W., Ong C. W., A. P. Balasuriya, Koh T. S. and Kow K. S. EOG based control of mobile assistive platforms for the severely disabled. In Proc. of the International Conference on Robotics and Biomimetics (ROBIO 2005), pages 490-494, 2005.

14. F. Mizuno, T. Hayasaka, K. Tsubota, S. Wada and T. Yamaguchi. Development of hands-free operation interface for wearable computer-hyper hospital at home. In Proc. of the 25th Annual International Conference of the Engineering in Medicine and Biology Society (EMBS 2003), pages 3740-3743, 2003.

15. D. W. Patmore and R. B. Knapp. Towards an EOG-based eye tracker for computer control. In Proc. of the 3rd International ACM Conference on Assistive Technologies (Assets 1998), pages 197-203, 1998.

16. A. Bulling, D. Roggen and G. Tröster. It's in Your Eyes - Towards ContextAwareness and Mobile HCI Using Wearable EOG Goggles. In Proc. of the 10th International Conference on Ubiquitous Computing (UbiComp 2008), 2008. in print - available from author.

17. C. Magerkurth, A. D. Cheok, R. L. Mandryk and T. Nilsen. Pervasive games: bringing computer entertainment back to the real world. Computers in Entertainment (CIE 2005), 3(3):4-4, 2005.

18. P. Isokoski, A. Hyrskykari, S. Kotkaluoto and B. Martin. Gamepad and Eye Tracker Input in FPS Games: Data for the First 50 Minutes. In Proc. of the 3rd Conference on Communication by Gaze Interaction (COGAIN 2007), pages 78-81, 2007.

19. M. Hayhoe and D. Ballard. Eye movements in natural behavior. Trends in Cognitive Sciences, 9:188-194, 2005.

20. M. Csíkszentmihályi. Flow: The Psychology of Optimal Experience. Harper Collins, New York, 1991.

21. S. P. Liversedge and J. M. Findlay. Saccadic eye movements and cognition. Trends in Cognitive Sciences, 4(1):6-14, 2000.

22. J. Skotte, J. Nøjgaard, L. Jørgensen, K. Christensen and G. Sjøgaard. Eye blink frequency during different computer tasks quantified by electrooculography. European Journal of Applied Physiology, 99(2):113-119, 2007.

23. P. P. Caffier, U. Erdmann and P. Ullsperger. Experimental evaluation of eyeblink parameters as a drowsiness measure. European Journal of Applied Physiology, V89(3):319-325, 2003. 